\title{
On Time Series Analysis for Repeated Surveys
}

\author{
Mohamed A. Ismail ${ }^{1, *}$, Hend A. Auda ${ }^{2}$, and Yehia Ahmed Elzafrany ${ }^{3}$ \\ ${ }^{1}$ Professor, Statistics Department, Cairo University \\ ${ }^{2}$ Associate Professor, Statistics Department, Helwan University \\ ${ }^{3}$ Statistical Advisor, National Centre for Statistics \& Information
}

A RT I C L I N F O
Article History
Received 06 Jul 2016
Accepted 04 Jul 2017
Keywords
Cross section surveys
ARMA models
State space
Unemployment rate
2000 Mathematics Subject
Classification
22E46, 53C35, 57S20

\section{INTRODUCTION}

Repeated surveys are usually used in economics and social science, by industry, government and research institutions to identify the characteristics of the population under study. It is often done at regular basis to monitor not only the level of some parameters of interest but also their changes in the intervals between surveys. It is also used to build up information on the trends of the phenomenon. The time series analysis for repeated surveys can be used to obtain more accurate estimation of the survey responses corresponding to the parameter of interest when compared to the traditional sample survey approach of separate parameter estimation at each time period (see [1]).

In Egypt, many surveys are repeated at fixed time intervals (monthly, quarterly, annually, or semiannually) such as labor force surveys conducted by the Egyptian Central Agency for Public Mobilization and Statistics (CAPMAS), and business surveys. Other surveys are repeated on an occasional basis. Examples include exit polls, and monitoring TV ratings. The time-series nature of these repeated surveys is seldom taken into account, which leads to lose the benefits of using the time series analysis of repeated surveys. The repeated nature of these surveys data creates a need for estimation procedures that combine information available from different times to produce the best estimates for the current mean or (percent estimators).

Using repeated surveys in estimating mean or percent of some indicators was subjected to many studies through the previous decades. Starting with Jessen [2], Patterson [3], Blight and Scott [4], Scott and Smith [5], Feder [6], Van den Brakel and Krieg [7], and many other studies tried to use different time series methods in repeated surveys. In Section 2, the literature of the time series methods used in repeated surveys is presented. A simulation study to compare between the last survey estimate and the time series estimator developed by Scott et al. [1] is provided in Section 3. In Section 4, an application of time series methods on the yearly Egyptian unemployment rate data obtained by the Egyptian CAPMAS (1980-2012) using the labor force survey is presented.

\section{TIME SERIES ANALYSIS OF REPEATED SURVEYS}

The time series repeated surveys estimators depend on the past information obtained from previous estimates to improve the current estimates. Time series repeated surveys estimators could have lower variance than the corresponding traditional estimators (see $[8,9])$. The improved time series repeated surveys estimators is no longer a function defined only on the sample at one time period $t$, but instead defined on all samples taken between two fixed time periods 1 and $t$. Thus, the estimator is derived from $t$ periods of data. Previous studies on

*Corresponding author. Email: mismail@feps.edu.eg 
analyzing repeated survey methods can be classified into two categories; the classical method (non-stochastic approach), and the time series methods (stochastic approach).

The pioneer work of the classical methods was that of Jessen [2] for the sample of two occasions. Yates [10] extended Jessen's work for the case of constant sample size and a fixed replacement fraction on each occasion. Patterson [3] generalized the results of Jessen to the case of sampling on $h$ occasions (surveys) with partial overlap of sampling units. Eckler [11] obtained a minimum variance estimate of the population value using the method of rotation sampling. Cochran [12] developed the optimum overlap for Patterson's [3] estimator when all samples are of equal size. Rao and Graham [13] used the rotations sample design to develop a unified finite population theory for composite estimators of the current levels. Singh [14] studied the effect of using multi-stage sample design on Patterson's estimator.

The time series approach can be a solution for problems of the classical methods in which any relationship between successive values of the population parameters is ignored completely, therefore using the classical methods requires the individual unit values be available to the analyst, however in many cases the individual units are not available for analysis, and the secondary analysis must be used, which in turn requires using the time series methods. For the time series methods, it can be classified into two categories; the ARMA method approach, and the state space approach.

Considerable interest has been shown in the methods of estimation for repeated surveys using time series analysis (ARMA method approach); Blight and Scott [4] discussed the effect of ignoring the relationship between successive values of the population parameters using a Bayesian approach. They also explored the effect of using such relationship in the estimation process.

Scott and Smith [5] used a more general approach than that used by Blight and Scott [4]. They assumed a stochastic model for the population means with a stationary process or a non-stationary process which can be reduced to a stationary form using differencing or subtracting deterministic components.

Scott et al. [1] extended the results of Scott and Smith [5] to surveys of complex designs, and applied their results on both overlapping and non-overlapping surveys. Some comparisons of Blight and Scott [4], Scott and Smith [5] approach with that of Patterson [3] using the mean square error (MSE) of the current mean was given by Jones [15]. Jones [16] derived a general form for the estimators derived by Blight and Scott [4], Scott and Smith [5], and Patterson [3] using the least square theory. The estimators of the population means obtained by Patterson, Blight and Scott, and Scott and Smith could be obtained from Jones's estimator using the generalized least square method. The problem of stationarity is also considered by Jones [16].

A new approach of the time series methods for repeated surveys is that of the state space approach. This approach consists of measurement, and transition equation. The population means are then estimated using the Kalman filter technique. Unlike the ARMA method approach, the state space approach can be useful in the cases where a small number of observations are used, or having a data with missing observations. Subsequent work for the state space approach by Tam [17], Rao et al. [18], Pfeffermann [19], Feder [6], Silva and Smith [20], Lind [21], Pfeffermann and Tiller [22], Sadik and Notodiputro [23], Van den Barkel and Krieg [24], and Krieg and Van den Barkel [25].

\section{SIMULATION STUDY}

\subsection{Simulation Design}

The simulation design in the current study is discussing two main cases:

Case 1: Correct specified time series models

Case 2: Miss-specified time series models

Under each case, we investigate the following factors:

1. The sampling variance $\left(S^{2}\right)$

The survey sampling errors were assumed to follow the normal distribution with zero mean and a sampling variance $\left(S^{2}\right)$, where the sampling variance takes the values $0.5,1$, or 2 .

2. Number of surveys $(t)$

Different number of surveys $t$ (the number of the sample means) were used to trace the efficiency of the following two estimators. The time series estimator obtained by Scott et al. [1] with the following equation:

$$
\hat{\hat{\theta}}_{t}=(1-\pi) \hat{\theta}_{t}+\pi \hat{Z}_{t-1}
$$

And the last survey estimator $\left(\hat{\theta}_{t}\right)$ obtained using the last value in the number of surveys $t$ using the following equation:

$$
\hat{\theta}_{t}=\theta_{t}+e_{t}, \quad e_{t} N\left(0, S^{2}\right)
$$


Table 1 The different scenarios for the simulation design.

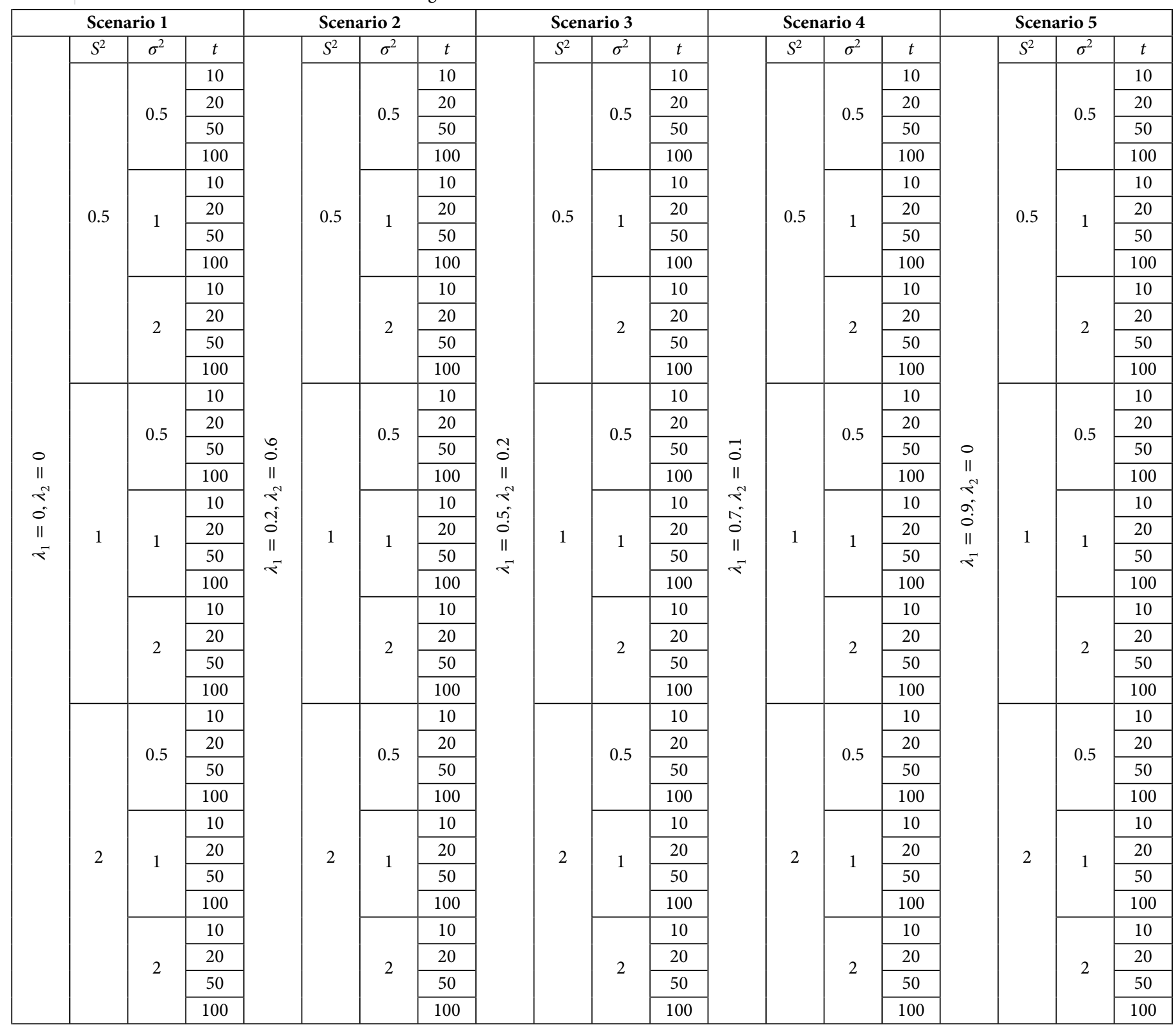

The numbers of surveys used into the simulation are 10,20,50, and 100, where 10 represents smallest number of surveys and 100 represent the largest number of surveys.

To check the effect of the series size, the selected number of surveys $t$ was created using the last $t$ values in the series of the sample means, so that the values for the sample means included in the smaller values for $t$, will be included in the larger values for $t$ (if $t=20$, then the sample means of the series of size $10(t=10)$ were included in the series).

3. The ARMA model coefficients

Different parameter values for the ARMA model coefficients $\left(\lambda_{\theta}\right.$ and $\left.\lambda_{e}\right)$ were considered to investigate the effect of parameter uncertainty on the efficiency of the time series estimator $(\lambda=0,0.2,0.5,0.7$, or 0.9$)$ for $\mathrm{AR}(1)$, and $\mathrm{MA}(1)$.

Based on the above design, we have all different scenarios given in Table 1.

\subsection{Simulation Steps}

1. Data is generated from $\operatorname{ARMA}(p, q)$ where 600 random values for the random error $\epsilon_{t}$ are firstly generated assuming that $\epsilon_{t} N\left(0, \sigma^{2}\right)$, where the variance takes the values $0.5,1$, or 2 .

2. The pseudo population means $\theta_{t}$ were created assuming that the initial values are zeroes. 
3. The first 500 values of the series are deleted to remove the initialization effect. For each of the factors mentioned in Table 1 , the process is repeated 10000 runs, and for every run the values of the two estimators; Eqs. (1) and (2) are recorded.

4. The MSEs and the confidence intervals for the two estimators are then computed to compare the efficiency of the two estimators.

5. The MSE is computed as averages across all other factors, for example to compute the MSE for $S^{2}=0.5$, we get the averages over all cases of $t$ and $\lambda$

\subsection{Simulation Results}

The results for the comparisons for the two estimators are presented first for the two cases (correct specified, and miss-specified) as an overall result, then the comparison is done for each of the factors described in Table 1.

\subsubsection{The Overall Results}

\section{Correct Specified Time Series Models}

Figure 1 displays the boxplot for the MSE of the two estimators using different time series models.

Based on Fig. 1, it can be noticed that the 1st quartile, the median, and the 3rd quartile of the MSE of the time series estimators are always less than those of the last survey estimator using the different Time Series Modeling TSM, which indicates the superiority of the time series estimator when compared to the last survey estimator.

Also, the boxplots of the time series estimator are taller than that of the last survey estimator as 50\% of the MSE of the time series estimators are between 0.30 and 0.64 for $\mathrm{AR}(1), 0.28$ and 0.68 for $\mathrm{AR}(2), 0.32$ and 0.80 for $\mathrm{MA}(1)$, and 0.32 , and 0.87 for $\mathrm{MA}(2)$ whereas $100 \%$ of the MSE of the last survey estimator are around 0.5 and 2 for the different time series models (this is logically true as the MSE of the last survey estimator is the same as the sampling variance $S^{2}$, and that may indicate that the simulation is correctly implemented). From Fig. 1, we conclude that the values of the MSE of the time series estimator are more homogeneous than that of the last survey estimator, and they are centered on small values of the MSE which reflects the efficiency of the time series estimator.

\section{Miss-specified Time Series Models}

Table 2 displays the MSE for the last and time series estimators using the different miss-specified time series models.

The comparison between the MSEs for the two estimators indicates that the MSE of $\left(\hat{\hat{\theta}}_{t}\right)$ is always less than that of $\left(\hat{\theta}_{t}\right)$ even when using a miss-specified time series models. the difference between the last survey and time series estimators is on average -0.78 when using the misspecified $\operatorname{AR}(2)$ for the correct $\operatorname{AR}(1)$, and even if using the misspecified MA(1), and MA(2), the MSE of the time series estimator is still lower than that of the last survey estimator (the difference reached to -0.33 and -0.36 respectively). Similar results can be found when using the different miss-specified time series models.

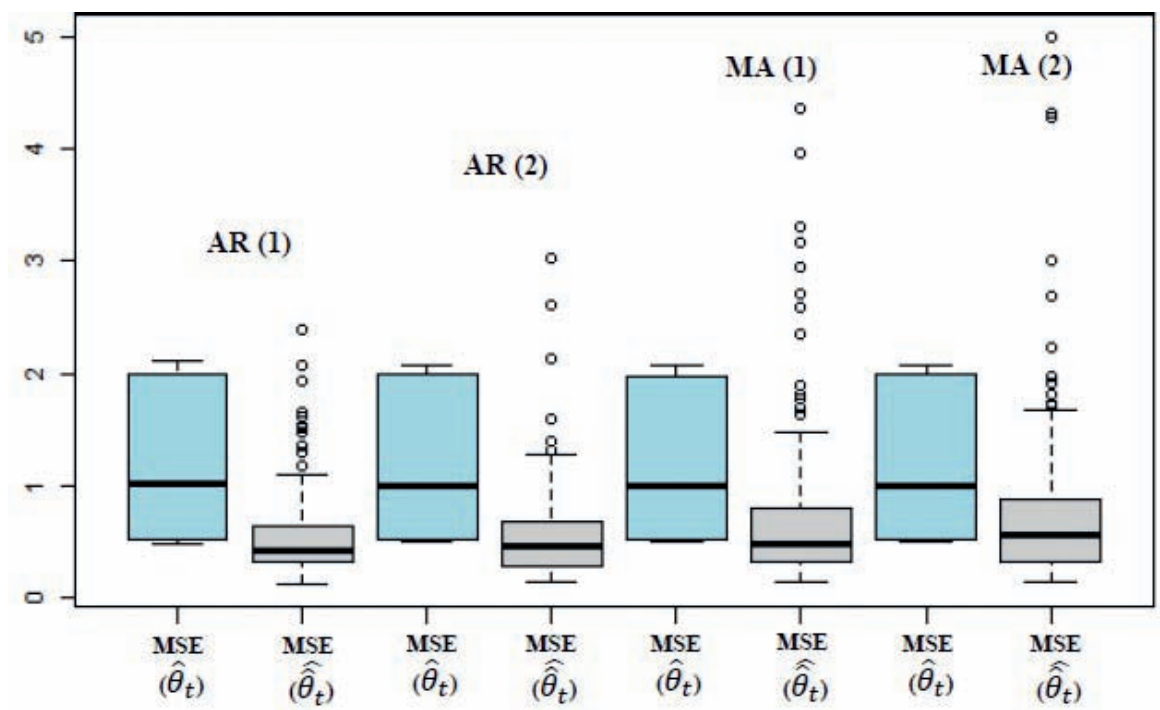

Figure 1 The box plot for the MSE of the two estimators using the different time series models. 
Table 2 The average difference between the MSE for the last estimator and time series estimators using different TSM.

\begin{tabular}{lcccc}
\hline Model & AR(1) Diff & AR(2) Diff & MA(1) Diff & MA(2) Diff \\
\hline AR(1) & -0.58 & -0.78 & -0.33 & -0.36 \\
AR(2) & -0.67 & -0.60 & -0.43 & -0.51 \\
MA(1) & -0.75 & -0.64 & -0.46 & -0.63 \\
MA(2) & -0.56 & -0.57 & -0.44 & -0.42 \\
\hline
\end{tabular}

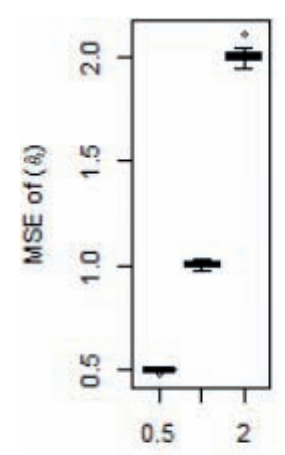

Sampling Variance

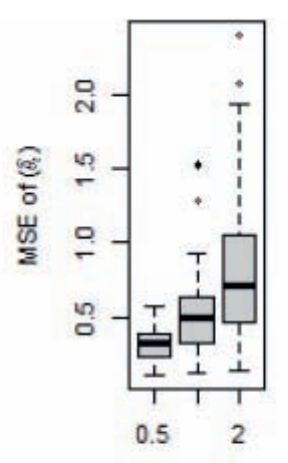

Sampling Variance

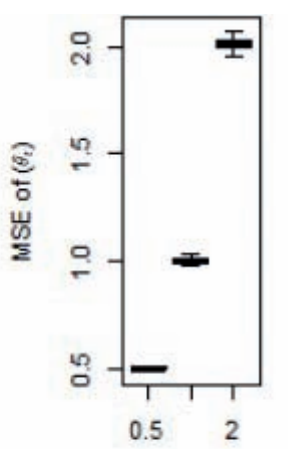

Sampling Variance

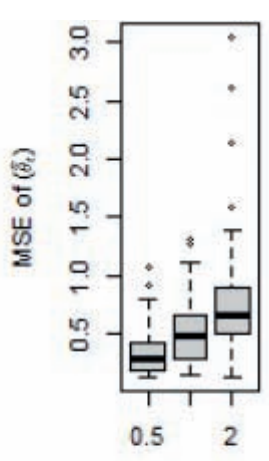

Sampling Variance AR (2)

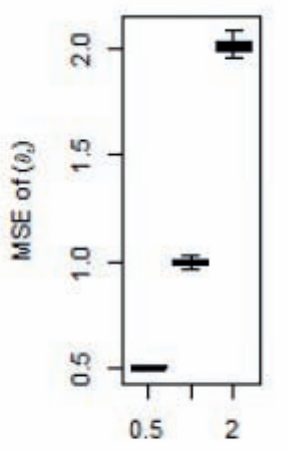

Sampling Variance

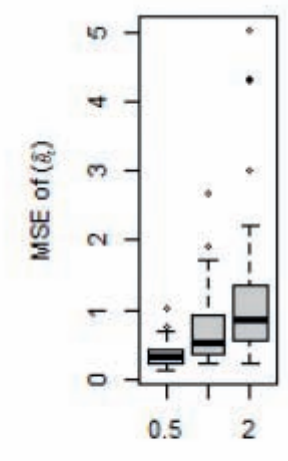

MA (2)

Figure 2 The MSE for the last and time series estimators using the different correct TSM according to the sampling variance.

\subsubsection{Results Based on Simulation Factors for Correct Specification Case}

\section{Sampling Variance $\left(\mathrm{s}^{2}\right)$}

First to be investigated is the sampling variance $S^{2}$. The results show that the values of the MSE of the time series and last survey estimators get larger when the sampling variance is larger. The superiority of $\left(\hat{\hat{\theta}}_{t}\right)$ to $\hat{\theta}_{t}$ has a positive relationship with $S^{2}$. These results are obtained for all used models; $\operatorname{AR}(1), \operatorname{AR}(2), \operatorname{MA}(1)$, and MA(2). Fig. 2 displays the boxplots for the MSE of the two estimators according to the sampling variance and using different time series models.

It seems from the boxplots that the MSEs of the last survey estimator are always around the values of the sampling variance $\left(S^{2}\right)$ which provides an evidence for the validity of the simulation (the MSE of the last survey estimator is supposed to equal to the sampling variance $\left(S^{2}\right)$ ). It can also be found that the 1st quartile, the median, and the 3rd quartile, of the MSE of the time series estimators are always less than those of the last survey estimator for all different values of the sampling variance $\left(S^{2}\right)$. 


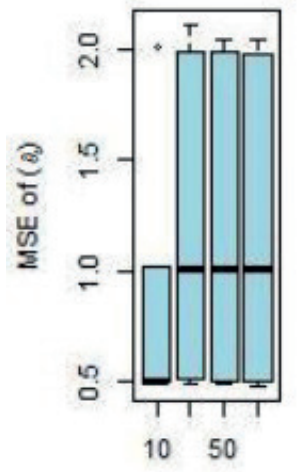

Number of Surveys

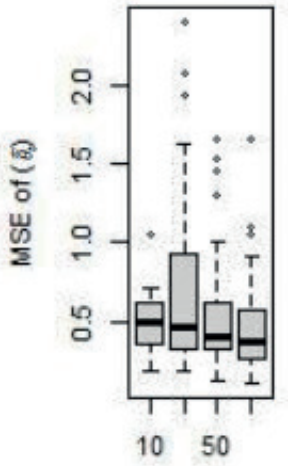

Number of Surveys

AR (1)

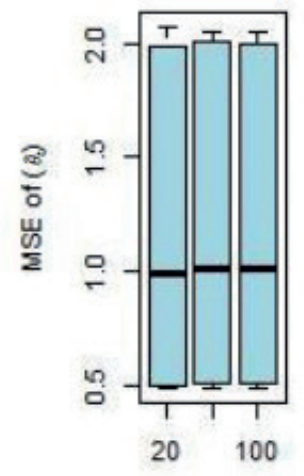

Number of Surveys

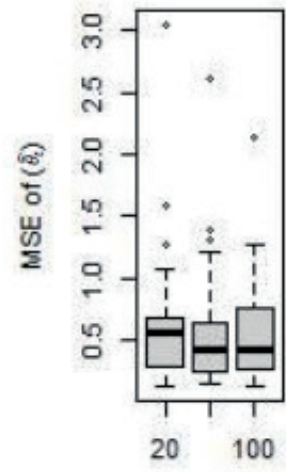

Number of Surveys

AR (2)

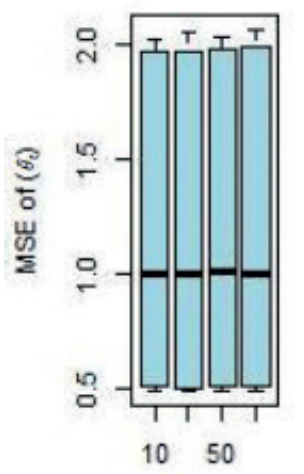

Number of Surveys

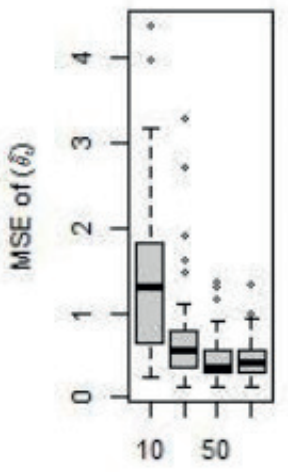

Number of Surveys

MA (1)

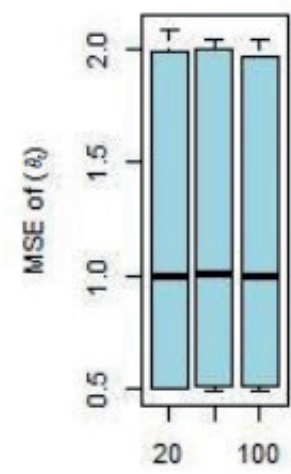

Number of Surveys

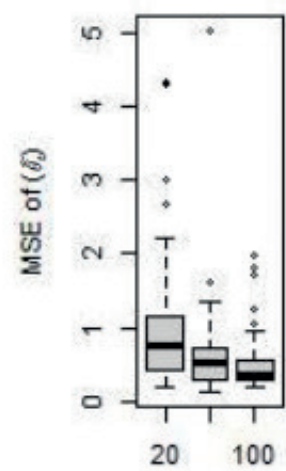

Number of Surveys

MA (2)

Figure 3 The MSE for the last and time series estimators using different correct TSM according to the number of surveys.

\section{The Number of Surveys $(t)$}

The second factor is the number of surveys $(t)$. It is clear that the superiority of $\left(\hat{\theta}_{t}\right)$ to $\hat{\theta}_{t}$ increases when the number of surveys increases. This result is valid for all used time series models (AR(1), AR(2), MA(1), and MA(2)). Fig. 3 displays the boxplots for the MSE of the last survey and time series estimators according to the different values of number of surveys using different TSM.

Figure 3 shows that the median of the MSE of the time series estimator nearly decreases when using larger values for the number of surveys $(t)$. It is also found that the first quartile, the median and the third quartile of the MSE of the time series estimator are always less than those of the last survey estimator for the different values of $(t)$. When using MA(1) model, it was found that the median of the MSE of the last survey estimator is less than that of the time series estimator when the number of surveys $(t=10)$. This implies that the time series estimator may suffer from the problem of model failure when using a small number of surveys as was indicated in the literature, see e.g., Tam [17].

\section{The Model Coefficient $(\lambda)$}

The analysis of the results according to the value of $\lambda$ doesn't detect any clear pattern for the relation between the MSE of $\left(\hat{\hat{\theta}}_{t}\right)$ and the different values of $\lambda$ for the used TSM. Fig. 4 displays the MSE for the last and time series estimators using the different time series models according to ARMA model coefficients.

As shown in Fig. 4, the 1st quartile, the median and the 3rd quartile of the time series estimator are always less than those of the last survey estimator using the different time series models, which reflects the superiority of the time series estimator. However, the values of the MSE of the time series estimator haven't any clear pattern for the different values of $\lambda$. 


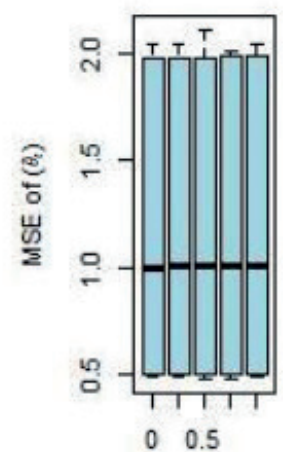

ARMA Model Coef

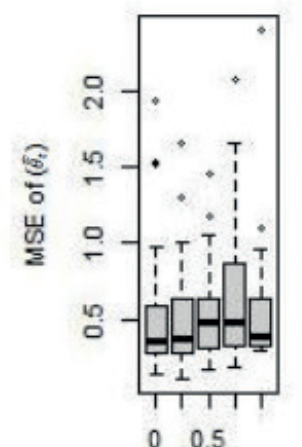

ARIMA Model Coef

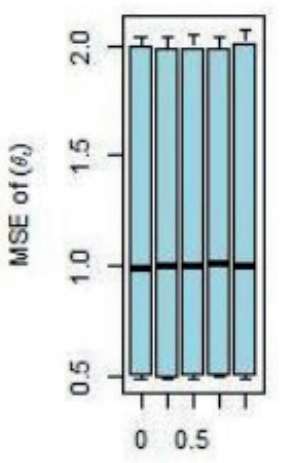

ARMA Model Coef

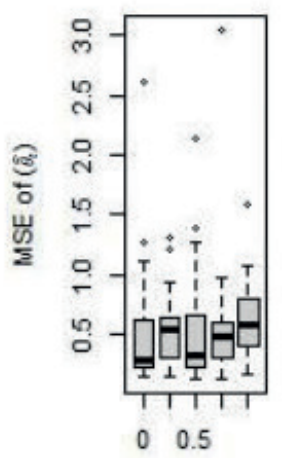

ARIMA Model Coef

AR (2)

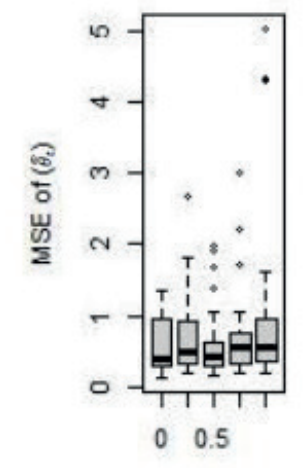

ARMA Model Coef

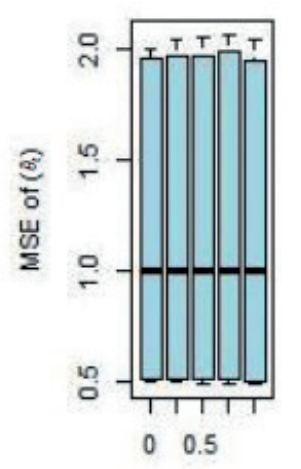

ARMA Model Coef

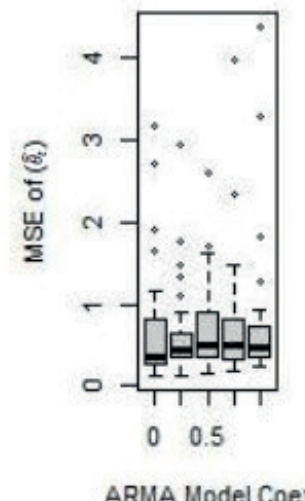

MA (1)

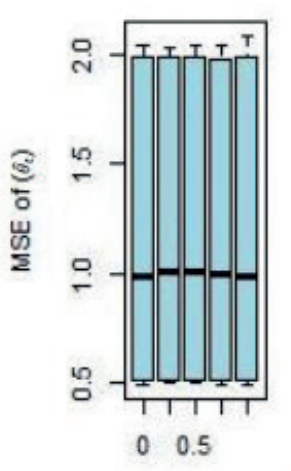

ARMA Model Coef

MA (2)

Figure 4 The MSE for the last and time series estimators using the different TSM according to ARMA model coefficients.

\subsubsection{Results Based on Simulation Factors for Miss-specified Case}

\section{Sampling Variance $\left(\mathrm{s}^{2}\right)$}

For the case of miss-specified time series models, the MSE of $\widehat{\hat{\theta}}_{t}$ is still less than that of $\hat{\theta}_{t}$, and whatever the value of the sampling variance $S^{2}$. Also the superiority of the time series estimator $\hat{\theta}_{t}$ to $\hat{\theta}_{t}$ still gets larger when using larger values for the sampling variance.

As shown from Table 3, the difference between the MSE of the last survey and the time series estimators using the miss-specified AR(2) instead of the correct $\operatorname{AR}(1)$ still increases with $S^{2}$ and reaches -1.44 for $S^{2}=2$ which is larger than the difference when $S^{2}=0.5$ and 1 (the difference $=-0.26$, and -0.66 respectively). Similar results can be found when using the other miss-specified models for AR( 1$)$ or the other miss-specified models for $\operatorname{AR}(2), \operatorname{MA}(1)$, and $\mathrm{MA}(2)$.

\section{The Number of Surveys ( $t)$}

Under the miss-specification case, the superiority $\hat{\hat{\theta}}_{t}$ to $\hat{\theta}_{t}$ is increased when using larger number of surveys. In terms of using small number of surveys for miss-specified TSM, the MSE of $\hat{\hat{\theta}}_{t}$ is affectedand can be more than that of $\hat{\theta}_{t}$. Table 4 shows the difference between the MSE of the last survey and time series estimators using miss-specified TSM and according to the different values of the used number of surveys.

From Table 4, there is a slight increase in the difference between the MSE of the last survey and time series estimators when using larger values of $t$ even if using miss-specified TSM. However, when using small number of surveys and miss-specified TSM, the efficiency of the time series estimator gets lower compared to that of the last survey estimator. The difference becomes $0.32,0.5$, and 0.31 when using the miss-specified MA(1) for the correct $\mathrm{AR}(1), \mathrm{AR}(2)$, and MA(2), respectively. (The time series estimator suffers from the problem of model failure as mentioned in the literature.) 
Table 3 The average difference between the MSE of $\hat{\hat{\theta}}_{\mathrm{t}} \& \hat{\theta}_{\mathrm{t}}$ acco rding to different TSM and the sampling variance.

\begin{tabular}{lccccc}
\hline Model & $\mathbf{s}^{\mathbf{2}}$ & AR(1) Diff & AR(2) Diff & MA(1) Diff & MA(2) Diff \\
\hline \multirow{3}{*}{ AR(1) } & 0.5 & -0.18 & -0.26 & -0.12 & -0.11 \\
& 1.0 & -0.48 & -0.66 & -0.27 & -0.24 \\
& 2.0 & -1.14 & $-\mathbf{1 . 4 4}$ & -0.63 & -0.73 \\
\hline \multirow{3}{*}{ AR(2) } & 0.5 & -0.18 & -0.15 & -0.14 & -0.16 \\
& 1.0 & -0.51 & -0.46 & -0.34 & -0.36 \\
& 2.0 & $-\mathbf{1 . 3 4}$ & -1.21 & -0.84 & -1.04 \\
\hline \multirow{3}{*}{ MA(1) } & 0.5 & -0.22 & -0.14 & -0.14 & -0.22 \\
& 1.0 & -0.60 & -0.47 & -0.30 & -0.57 \\
& 2.0 & $-\mathbf{1 . 4 3}$ & $-\mathbf{1 . 4 1}$ & -0.94 & -1.11 \\
\hline & 0.5 & -0.18 & -0.26 & -0.12 & -0.11 \\
MA(2) & 1.0 & -0.16 & -0.22 & -0.16 & -0.14 \\
& 2.0 & $-\mathbf{1 . 1 4}$ & -1.09 & -0.80 & -0.83 \\
\hline
\end{tabular}

All bolded numbers are significant at 0.05 .

Table 4 The average difference between the MSE of $\widehat{\hat{\theta}}_{t} \& \hat{\theta}_{t}$ according to different TSM and the number of surveys.

\begin{tabular}{cccccc}
\hline Model & $\mathbf{s}^{2}$ & AR(1) & AR(2) & MA(1) & MA(2) \\
\hline & 10 & Diff & Diff & Diff & Diff \\
& 20 & -0.26 &.-- & $-\mathbf{0 . 3 2}$ &.-- \\
AR(1) & 50 & -0.48 & -0.72 & -0.58 & -0.06 \\
& 100 & -0.63 & -0.84 & -0.50 & -0.51 \\
& 10 & -0.71 & -0.78 & -0.51 & -0.49 \\
\hline & 20 & -0.21 &.-- & $-\mathbf{0 . 5 0}$ &.-- \\
AR(2) & 50 & -0.60 & -0.52 & -0.62 & -0.38 \\
& 100 & -0.76 & -0.63 & -0.73 & -0.51 \\
& 10 & -0.71 & -0.65 & -0.74 & -0.64 \\
\hline & 20 &.-- &.-- & -0.50 & -0.33 \\
MA(1) & 50 & -0.64 & -0.23 & -0.43 & -0.81 \\
& 100 & -0.83 & -0.71 & -0.69 & -0.78 \\
\hline & 10 & -0.78 & -0.78 & -0.71 & -0.10 \\
AR(1) & 20 & -0.19 &.-- & -0.12 \\
& 50 & -0.53 & -0.29 & -0.58 & -0.51 \\
& 100 & -0.72 & -0.65 & -0.78 & -0.63 \\
\hline
\end{tabular}

All bolded numbers are significant at 0.05 .

\section{THE ANNUAL UNEMPLOYMENT RATE}

Our two estimators are applied for the series of the annual unemployment rate. This series ran from the period of 1980 until the end of the year 2012. ${ }^{1}$ The source of the data is the Egyptian Labor Force Surveys conducted by the Egyptian CAPMAS. This survey was first conducted in November 1957, and since then the survey has been conducted but in irregular periodicity as it was conducted annually in times and biannually or quarterly in others. It aims at measuring the Egyptian civilian labor force and its characteristics, measuring the level of employment in different geographic areas of the republic, and measuring the geographic distribution of the employed population according to many characteristics like gender, age, education, etc. The survey sample is a two-stage stratified cluster sample, and its size is about 21352 households for each round allocated among governorates and their urban/rural components in proportion to size (Fig. 5).

Following Box and Jenkins procedures to estimate the model for the yearly unemployment rate resulted in an ARIMA (1,2,1) model. This model achieved all the assumptions and seems to be efficient to represent the data. Hence the estimated values for $\pi$ and $\hat{Z}_{(t-1)}(1)$ can be derived as shown in Table 5.

The data during the period 1985 until 1989 were missing, and they were replaced using the linear interpolation method. 


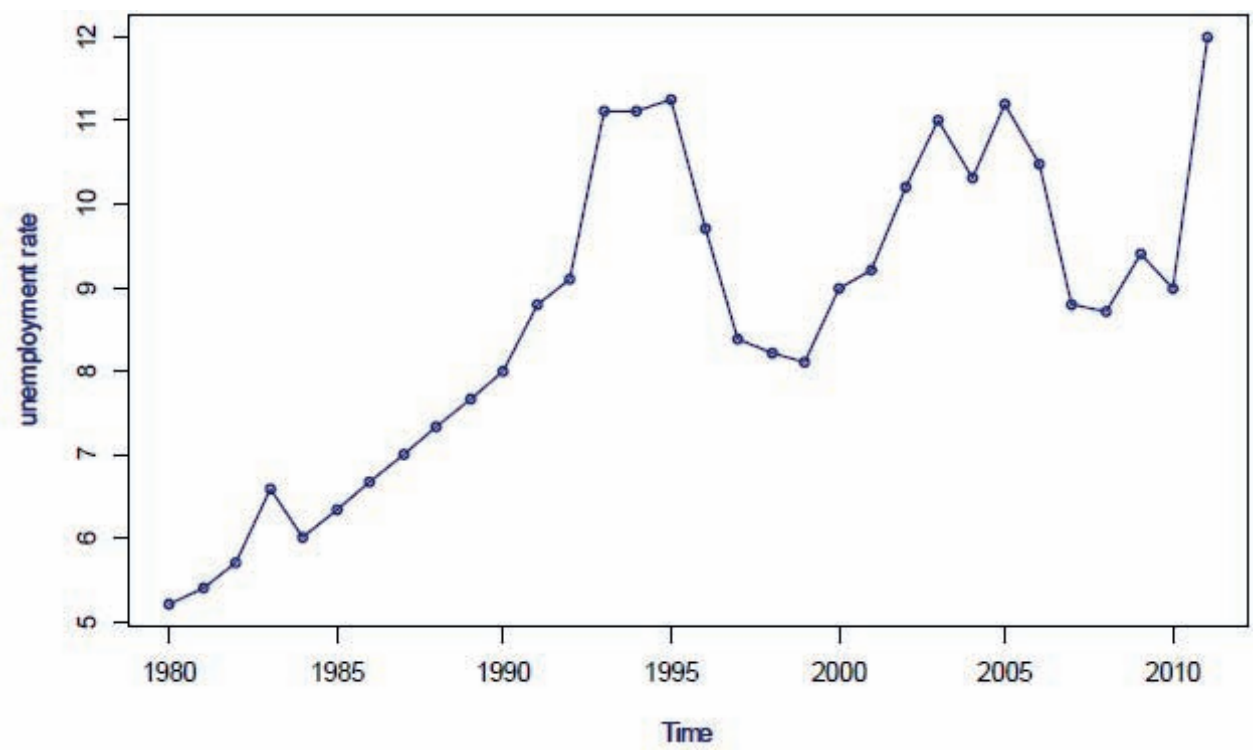

Figure 5 The time series plot for the Egyptian unemployment rate during the period 1980-2012.

Table 5 Comparison of acoustic for frequencies for piston-cylinder problem.

\begin{tabular}{cc}
\hline The factor & The estimated value \\
\hline$\hat{\sigma}^{2}$ & 1.510 \\
$S^{2}$ & 1.005 \\
$\pi$ & 0.666 \\
$\hat{\theta}_{t}$ & 12.700 \\
$\hat{\hat{\theta}}_{t}$ & 12.966 \\
\hline
\end{tabular}

The unemployment rate in 2012 records $13 \%$ using the time series estimator compared to $12.7 \%$ using the last survey estimator as shown in Table 1 . This represents only $0.3 \%$ underestimation of the value of the unemployed people reached by the labor force survey. The gains due to the time series method depend on the value of $\frac{S^{2}}{\sigma^{2}}$ which is $6.7 \%$.

A 95\% confidence intervals for the mean of the unemployment rate of $\hat{\theta}_{t}$ and $\hat{\hat{\theta}}_{t}$ are also constructed. The variance of the time series estimator is lower than that of the last survey estimator as it is 1.005 for the time series estimator, compared to 1.510 for the last survey estimator. This is reflected on the two confidence intervals. The comparison shows that the limits of the confidence interval using the time series estimator are between $11.830 \%$ and $14.102 \%$ which is much smaller than the confidence interval using the last survey estimator as it is between $3.965 \%$ and $21.435 \%$. The efficiency of the time series estimator is shown in the following Fig. 6.

\section{CONCLUSION}

The analysis of repeated surveys using time series methods is seldom taken into account. The estimation of the mean of the phenomena usually depends on the last survey, although it is more efficient to use the time series analysis in the estimation process as the time series for repeated surveys estimators could have lower variance than the corresponding traditional estimators. This was confirmed using the simulation study which indicated that the MSE of the time series mean $\hat{\hat{\theta}}_{t}$ is always less than that of the last survey estimator $\hat{\theta}_{t}$ using the different time series models.

It is shown from the current study that the values of the 2 MSE's get larger when the sampling variance is larger, and also the superiority of $\hat{\hat{\theta}}_{t}$ to $\hat{\theta}_{t}$ has a positive correlation with the value of $S^{2}$. For the different number of surveys, it is also proved that the superiority of $\hat{\hat{\theta}}_{t}$ to $\hat{\theta}_{t}$ gets larger when the number of surveys is larger. However when using different values for $\lambda$, there is a random pattern between these values and the MSE of $\left(\hat{\hat{\theta}}_{t}\right)$ using the different time series models. The results also show that when using miss-specified time series models, the MSE of the time series estimator is always less than that of the last survey unless a small number of surveys are used. These results are supported using the practical example of the unemployment rate which indicated the efficiency of the time series method in estimating the unemployment rate than using the last survey estimator as its confidence interval and hence the variance are very small compared to that of the last survey estimator. 


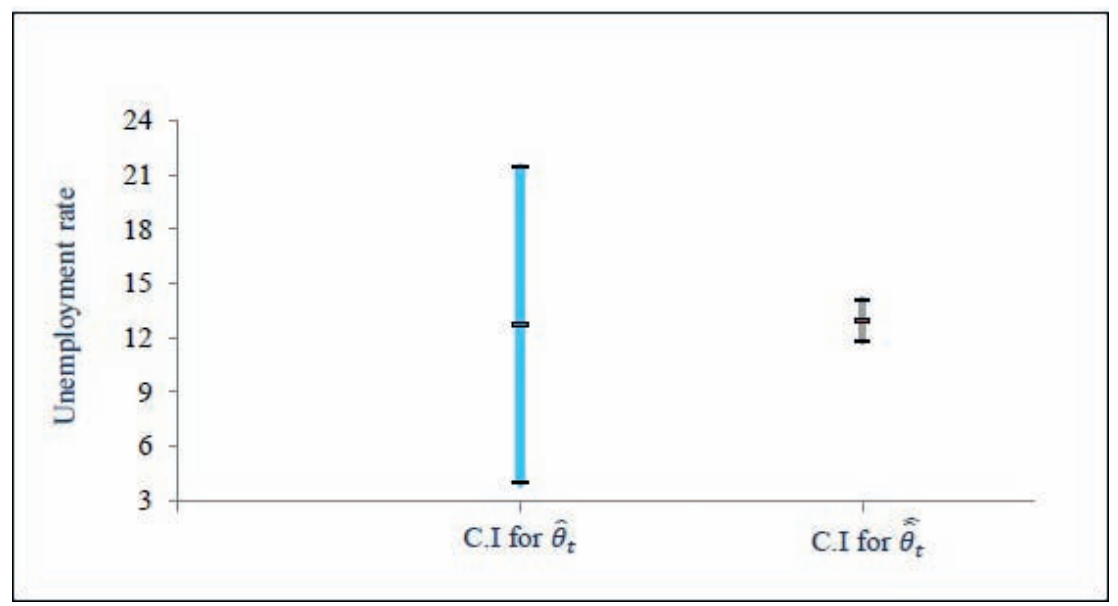

Figure 6 A 95\% confidence interval for the time series and last survey estimators using the unemployment rate.

\section{CONFLICT OF INTEREST}

Interest time series analysis \& repeated surveys.

\section{REFERENCES}

1. A.J. Scott, T.M.F. Smith, R.G. Jones, Int. Stat. Rev. 45 (1977), 13-28.

2. R.J. Jessen, Lowa Agric. Exp. Sta. Res. Bull. (1942), 304.

3. H.D. Patterson, J. Royal Stat. Soc. B. 12 (1950), 241-255.

4. B.J.N. Blight, A.J. Scott, J. Royal Stat. Soc. B. 35 (1973), 61-68.

5. A.J. Scott, T.M.F. Smith, J. Am. Stat. Assoc. 69 (1974), 674-678.

6. M. Feder, Stat. Neerl. 55 (2001), 182-199.

7. J.A. Van den Brakel, S. Krieg. Estimation of the monthly unemployment rate through structural time series modelling in a rotating panel design, Research paper, Statistics Netherlands, Heerlen, 2009.

8. D. Steel, G. Mclaren. Design and analysis of repeated surveys, Center for Statistical and Survey Methodology. University of Wollongong 2008. pp $8-13$.

9. S.J. Haslett, Victoria University of Wellington, 1986.

10. F. Yates, 3rd Edition, Charles Griffin and Co., London, 1960.

11. A.R. Eckler. Ann. Math. Stat. J. 26 (1955), 664-685.

12. W. G. Cochran 2nd. Aufl. John Wiley and Sons, New York, London 1963.

13. J.N.K. Rao, J.E. Graham, J. Am. Stat. Assoc. 59 (1964), 492-509.

14. D. Singh, J. Am. Stat. Assoc. 63 (1968), 99-112.

15. R.G. Jones, Aust. J. Stat. 21 (1979), 45-56.

16. R.G. Jones, J. Royal Stat. Soc. B. 42 (1980), 221-226.

17. S.M. Tam, Int. Stat. Rev. 55 (1987), 63-73.

18. J.N.K. Rao, K.P. Srinath, B. Quenneville, Paper presented at the International Symposium on Panel Surveys, Washington, D.C., November, 1986.

19. D. Pfeffermann, J. Bus. Econ. Stat. 9 (1991), 163-175.

20. D.B.N. Silva, T.M.F. Smith, Stat. Can. 27 (2001), 205-215.

21. J.T. Lind, Econom. J. 9 (2005), 1-10.

22. D. Pfeffermann, R. Tiller, J. Am. Stat. Assoc. 101 (2006), 1387-1397.

23. K. Sadik, and K.A. Notodiputro. A State Space Model in Small Area Estimation. ICCS-IX, Malaysia 2007. pp 658-662.

24. S. Krieg, J. A. Van den Barkel, Journal of Computational Statistics and Data Analysis, 56 (2012), 2918-2933.

25. S. Krieg, J.A. Van den Barkel, J. Comp. Stat. Data Anal. 56 (2012), 2918-2933. 